\title{
BENIGN PROLIFERATING PILAR TUMOUR OF THE ELBOW - A RARE CASE REPORT
}

\begin{tabular}{|l|lll}
\hline Plastic Surgery &
\end{tabular}

Jai Durairaj Paramasivam

Surya Rao Rao
Venkata Mahipathy*

Shanthini Vaitheeswaran

Sudharshan Srinivasa Murthy

\section{Dr. Jayaganesh Parthasarathy}

Assistant Professor, Dept. of Paediatric Surgery, Saveetha Medical College \& Hospital, Thandalam, Kanchipuram Dist. - 602105, Tamilnadu, India

Professor \& Head, Dept. of Plastic \& Reconstructive Surgery, Saveetha Medical College \& Hospital, Thandalam, Kanchipuram Dist. - 602105, Tamilnadu, India*Corresponding Author

Resident, Dept. of General Surgery, Saveetha Medical College \& Hospital, Thandalam, Kanchipuram Dist. - 602105, Tamilnadu, India

Resident, Dept. of General Surgery, Saveetha Medical College \& Hospital, Thandalam, Kanchipuram Dist. - 602105, Tamilnadu, India

Associate Professor of Pathology, Saveetha Medical Colege \& Hospital, Thandalam, Kanchipuram Dist. - 602105, India.

\section{ABSTRACT}

Benign proliferating pilar tumour also known as proliferating trichilemmal cyst is a rare tumor usually occurring on the head and neck region of elderly women and its pathognomonic feature is trichilemmal keratinization. Here, a 65 year old elderly female presented to us with a benign proliferating pilar tumour of the right elbow for the past 6 months for which wide local excision and a local flap was used to cover the post excisional defect. This should be differentiated from trichilemmal cyst as it has potential for malignant transformation. Thus, complete excision is recommended for all benign proliferating variants owing to their potential for locally aggressive behavior and malignant transformation

\section{KEYWORDS}

Benign, Rare, Surgery, Trichilemmal

\section{Introduction}

Proliferating pilar tumour, also known as trichemmal cyst, is a rare benign skin neoplasm with outer root sheath differentiation.(1) This tumour was first described by Wilson-Jones in 1966 as a lesion with a histologic capacity to mimic a squamous cell carcinoma. There have different terminologies used to describe this tumor like pilar tumor, proliferating pilar cyst, giant hair matrix tumor, hydatidiform keratinous cyst, trichochlamydocarcinoma, and invasive hair matrix tumor.(2) It usually occurs on the scalp of elderly women and its histological hallmark is the presence of trichilemmal keratinization.(2)

\section{Case Report}

A 65 year old elderly lady presented to us with a swelling in the left elbow for the past 6 months. It was spontaneous in onset and gradually progressive to the present size. She gives history of occasional pain and bleeding on touch. She is a known case of ischemic heart disease, chronic obstructive pulmonary disease and diabetes mellitus for the past 10 years. On examination, a $4 \times 3 \mathrm{~cm}$ ulceroproliferative lesion is seen in the left elbow with vitilgo patches in the surrounding skin. (Fig. 1)

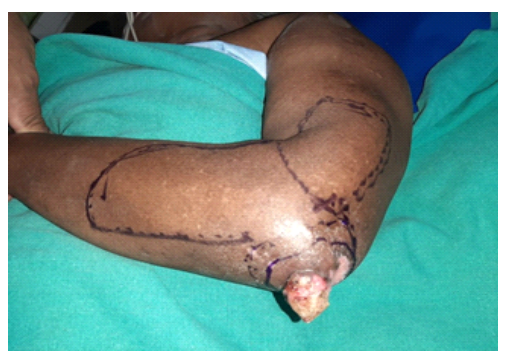

Fig. 1 - Clinical photograph showing the lesion in left elbow

\section{Discussion}

It has been observed that these tumours may arise de novo.(3) In most of cases, they occur on the scalp and the remaining occurring mainly on the back. There is an increased female predominance of 6:1, mostly in elderly women.(4,5) It generally starts as a subcutaneous nodule and gradually grows into a large, solitary, well-circumscribed mass. Sometimes, it may become ulcerated and infected and may resemble squamous cell carcinoma.(2) It can be differentiated from squamous cell carcinoma by the sudden onset of keratinization, trichilemmal differentiation, the presence of clear cells resulting in glycogen storage and most importantly, a sharp demarcation without infiltration into surrounding structures.(6). PTT is usually benign and rarely may undergo malignant transformation in a step-wise manner starting with an adenomatous stage of the trichilemmal cyst to an epitheliomatous stage of the PTT evolving into the carcinomatous stage of the malignant PTT, which is main consideration in the differential diagnosis.(7) The distinguishing feature of the malignant tumor is the addition of a frankly invasive component that may retain a clear cell pattern with classical trichilemmal keratinization. Nuclear and mitotic activities are variable. Vascular and/or perineural invasion may also be observed.(5,6) Thus, complete excision is recommended for all benign proliferating variants owing to their potential for locally aggressive behavior and malignant transformation. In a study by Ye J et al, 76 cases of proliferating pilar tumours were divided into three groups on the degree of stromal invasion and the level of cellular atypia: benign, low- and high-grade malignant. Group I included those patients who showed no infiltration of surrounding stroma and minimal nuclear atypia; they showed trichilemmal keratinization, stromal invasion with a mononuclear infiltrate of plasma cells and lymphocytes, and dystrophic calcification and were considered as benign tumors. Group II tumors were composed of multiple lobulated and bosselated expansive masses of squamous epithelium separated by loose edematous stroma and filled centrally with homogeneous acellular eosinophilic material representing amorphous debris and pilar keratin. In addition, the squamoid tumor cells manifested large, hyperchromatic nuclei with irregular nuclear membranes surrounded by abundant eosinophilic cytoplasm with a lack of marked cytologic atypia.Foci of single cell necrosis and abrupt keratinization were identified. Cords of atypical squamous epithelium extended into the surrounding dermis, with a desmoplastic stromal response. A minimal to moderate infiltrate of mononuclear inflammatory cells was identified as well. Group III tumors were invasive; they were cytologically anaplastic and were therefore considered malignant.(8)

\section{Conclusion}

Benign proliferating pilar tumours are commonly found on the scalp and back, but should be also kept in mind when similar clinical picture presents elsewhere in the body. Hence, a thorough history and clinical evaluation along with biopsy of the lesion will help us to clinch the diagnosis and treat it at the earliest as it is known for malignant transformation in a few cases.

The lesion was free from the underlying bone and there was no regional lymphadenopathy. An edge wedge biopsy revealed a 
diagnosis of a benign proliferating pilar tumour. We planned for surgical excision of the lesion along with the vitiligo patches and cover the defect with a local skin flap. Under axillary block and tourniquet control, the lesion was excised and the specimen was sent for histopathology. (Fig. 2, 3)
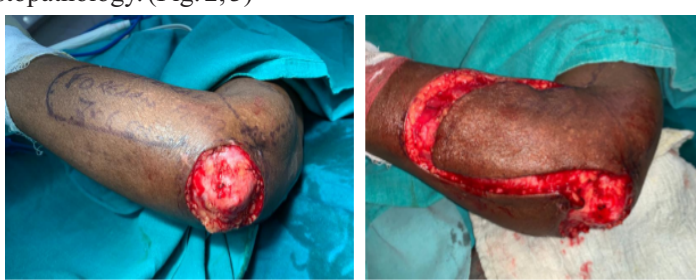

Fig. 2 - Picture after tumour excision

Fig. 3 - Local flap raised from forearm

The defect was covered with a fasciocutaneous flap raised from the proximal forearm. (Fig. 4)

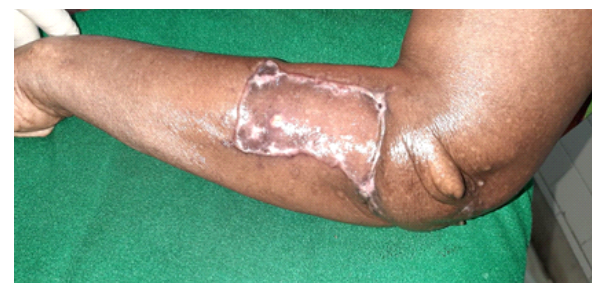

Fig. 4 - Well settled flap left elbow and skin graft in the flap donor site

The secondary raw area was covered with SSG harvested from the left thigh under local anaesthetic field block. The post-operative was uneventful and the patient was discharged on the 5th post-operative day. Histopathology showed a nodular circumscribed lesion with thick bands of stratified squamous epithelium showing trichilemmal-type extensive keratinisation and keratin pearls emanating from epidermis suggestive of a benign proliferating pilar tumour. (Fig. 5,6)

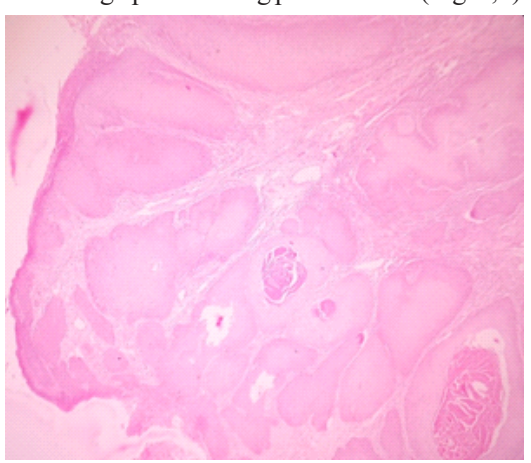

Fig. 5 - Low power view showing skin with neoplasm beneath composed of lobules of squamoid cells.

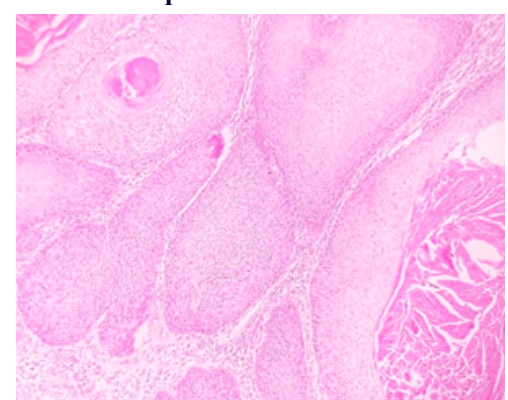

Fig. 6 - High power view showing squamoid cells with keratin pearls.

\section{References}

1. Leppard BJ, Sanderson KV. The natural history of trichilemmal cysts. Br J Dermatol 1976;94:379-90.

2. Brownstein MH, Arluk DJ. Proliferating trichelemmal cyst: A simulant of squamous cell carcinoma. Cancer 1981;48:1207-14.

3. Poiares Baptista A, Garcia E Silva L, Born MC. Proliferating trichilemmal cyst. J Cutan Pathol 1983;10:178-87.

4. Morgan RF, Dellon A, Hoopes JE. Pilar tumors. Plast Reconstr Surg 1979;63:520-4.
5. Satyaprakash AK, Sheehan DJ, Sangüeza OP. Proliferating trichilemmal tumors: A review of the literature. Dermatol Surg. 2007;33:1102-8

Chikhalkar S, Garg G, Gutte R, Khopkar U. Sebaceous carcinoma of scalp with proliferating trichilemmal cyst. Indian Dermatol Online J 2012;3:138-40.

7. Rao S, Ramakrishnan R, Kamakshi D, Chakravarthi S, Sundaram S, Prathiba D Malignant proliferating trichilemmal tumour presenting early in life: An uncommon feature. J Cutan Aesthet Surg 2011;4:51-5.

8. Ye J, Nappi O, Swanson PE, Patterson JW, Wick MR. Proliferating pilar tumors: A clinicopathologic study of 76 cases with a proposal for definition of benign and malignant variants. Am J Clin Pathol. 2004;122:566-74. 